\title{
Criminologie
}

\section{Le procès criminel : éléments d'une approche socio-juridique de la procédure pénale}

\section{Pierre Robert}

Volume 15, numéro 1, 1982

Droit et justice

URI : https://id.erudit.org/iderudit/017148ar

DOI : https://doi.org/10.7202/017148ar

Aller au sommaire du numéro

Éditeur(s)

Les Presses de l'Université de Montréal

ISSN

0316-0041 (imprimé)

1492-1367 (numérique)

Découvrir la revue

Citer cet article

Robert, P. (1982). Le procès criminel : éléments d'une approche socio-juridique de la procédure pénale. Criminologie, 15(1), 21-37.

https://doi.org/10.7202/017148ar d'utilisation que vous pouvez consulter en ligne.

https://apropos.erudit.org/fr/usagers/politique-dutilisation/ 


\section{LE PROCĖS CRIMINEL : ÉLEMENTS D'UNE APPROCHE SOCIO-JURIDIQUE DE LA PROCEDURE PENALE Pierre Robert*}

Une description du processus judiciaire présente toujours le risque d'une répétition aride de notions plus ou moins connues. Bien qu'un tel exercice conserve son mérite, il apparaît opportun de chercher au-delà de la lettre de la loi et de s'interroger sur les fondements premiers de ces règles et sur les objectifs qu'elles poursuivent.

L'observation de l'institution en action permet ensuite la critique de la règle de droit en ce qu'elle réalise ou non son objet.

La description académique du processus judiciaire pénal apparaît donc ici comme l'occasion d'une exploration épiphénoménologique de l'institution. Dans cette perspective, le procès, avec ses "dessous " prend un éclairage nouveau voire même inattendu. Quant à la règle de droit, cette approche est susceptible à la fois d'en faire découvrir les fondements et la nécessité, mais aussi les faiblesses et parfois même les limites.

Le procès pénal représente un phénomène sociologique complexe comportant des dimensions interdépendantes d'ordre politique et juridique comme psychologique et social. Tous les éléments de ces dimensions n'ont pas la même importance et surtout ne sont pas toujours aisément perceptibles. Ainsi les structures juridiques de l'instance judiciaire et les nombreuses règles de preuve sont évidemment apparentes et s'imposent généralement avec force. Par contre les préjugés sociaux, l'incompréhension ou l'humanisme sont également des éléments qui jouent dans le procès mais dont il est difficile de saisir la portée et même l'existence.

Par rapport à l'ordre juridique et jusqu'à un certain point l'ordre politique, les autres dimensions apparaissent essentiellement comme des épiphénomènes de la structure légale de base. Cette dernière les tolère et même les prévoit partiellement par l'accord des nombreuses discrétions prévues par la loi. La plus connue demeure sans doute l'énorme discrétion du juge sur la sentence. Nous y reviendrons. Par contre d'autres manifestations sont strictement interdites comme le préjugé ou le parti pris ouvert pour ou contre l'accusé ou un témoin. Les règles de récusation du juge ou les motifs d'oc-

* Avocat, substitut du procureur général du Québec et responsable de la coordination de la recherche et de la documentation. 
troi d'un bref de prohibition pour préjugé du tribunal illustrent les limites que le droit impose à certains épiphénomènes. Cependant la présence même de ces règles attestent de l'existence d'éléments psychologiques ou sociaux latents. La loi en réprime les manifestations les plus iniques, mais qu'en est-il des autres?

Enfin d'autres éléments qui composent le procès ne font souvent l'objet d'aucune attention juridique. La compétence ou l'incompétence des participants, la routine, la bureaucratie constituent autant d'éléments qui peuvent influer, à des degrés divers, sur l'issue d'un procès.

Non seulement ces éléments ne sont pas aisément perceptibles dans le déroulement d'une instance judiciaire mais ils ne s'y manifestent pas toujours et de la même manière.

Nous tenterons au cours de cette exploration d'en cerner les manifestations les plus évidentes à l'occasion des diverses étapes du procès.

\section{NATURE ET PRINCIPES FONDAMENTAUX DU PROCÉS}

D'un point de vue politique, le procès est, à la base, la confrontation d'un individu au corps social et à l'État. C'est l'occasion structurée de l'affirmation des valeurs sociales dominantes à l'encontre d'un comportement individuel contraire, à première vue, à ces valeurs.

Dès le départ, il y a donc déséquilibre : d'une part, tout le poids des valeurs d'une majorité ${ }^{1}$ allié à toute la force de l'appareil d'État et, d'autre part, un comportement délinquant et un individu déjà minoritaire.

L'équilibre est relativement rétabli par le droit. Cependant malgré cet ajustement ${ }^{2}$ il ne faut pas perdre de vue la précarité foncière de l'individu accusé surtout lorsqu'il est détenu.

Ce dernier, même récidiviste, est face à l'inconnu qui souvent se transforme pour lui en une certitude mentale : la réclusion. On entend parfois des propos de ces récidivistes pour qui l'issue du procès se règle au moment de l'arrestation avec la fermeture de la porte à verrouillage automatique de la voiture de patrouille. Une telle attitude dénote un désarroi symptomatique. Sans généraliser cet exemple extrême, il faut constater que la détention du prévenu contribue à le mettre dans une situation d'infériorité. Ceci se manifeste lors du procès par des détails qui peuvent parfois lui être préjudiciables.

1. Ce qui n'est pas une certitude pour toutes les infractions.

2. Qui est le fruit dans les systèmes occidentaux de plusieurs siècles de combats pour la justice et la civilisation. 
Par exemple l'individu dans le box des accusés est identifié par les témoins beaucoup plus facilement que l'individu mêlé au public de la salle d'audience. L'apparence négligée du détenu peut également constituer une situation désavantageuse qui influence plus ou moins l'opinion qu'on peut se faire de lui. Enfin le simple fait de la détention laisse planer une aura de suspicion malgré la présomption d'innocence. Cela se traduit également en pratique d'une part par une fréquence plus grande de plaidoyer de culpabilité pour l'accusé-prévenu que pour l'accusé en liberté et d'autre part par une certaine propension à imposer une peine d'incarcération à l'individu déjà détenu. C'est la solution de facilité. Évidemment ces constatations ne sont pas des règles absolues. Loin de là, fort heureusement! Néanmoins ce serait de l'aveuglement que de vouloir ignorer ces conséquences d'une détention avant procès qui par ailleurs a ses propres justifications.

L'infériorité de l'accusé est aussi accentuée par l'organisation judiciaire qui à l'instar de toute l'organisation gouvernementale projette chez le simple citoyen une image, parfois kafkaïenne de la bureaucratie. Les tribunaux, le ministère public, les forces de l'ordre sont autant de rouages qui peuvent créer un sentiment, lorsque ce n'est pas une véritable sensation, d'écrasement chez l'individu. Le droit, en général, élimine la possibilité de l'écrasement réel. En fait dans une perspective politique, la règle de droit constitue un frein à l'autorité arbitraire de l'État. C'est là du moins son objectif.

En effet, le principe juridique atténue fortement la confrontation inégale entre l'individu et l'État. Ainsi la loi prévoit des droits fondamentaux pour tout individu. Parmi ceux-ci le droit à l'égalité devant la loi de ne pas être soumis à l'arbitraire et d'avoir un avocat sont codifiés dans la Déclaration canadienne des droits de l'homme. De plus l'État favorise l'obtention aisée d'un avocat pour l'accusé. La loi de l'Aide juridique procède d'une volonté politique claire à cet égard. On pourrait certes donner d'autres exemples de règles juridiques qui visent à rétablir l'équilibre entre l'État et le citoyen. Nous aurons l'occasion d'en examiner plus en détail quelques-unes. Qu'il suffise pour l'instant d'insister sur la plus fondamentale de toutes, la présomption d'innocence.

La présomption d'innocence demeure certainement la manifestation la plus connue du principe de la légalité. L'article $5 \mathrm{du}$ Code criminel précise la portée de cette règle :

Article 5(1)

Lorsqu'une disposition crée une infraction et autorise l'impo- 
sition d'une peine à son égard :

a) une personne est réputée innocente de cette infraction tant qu'elle n'a pas été déclarée coupable;

et

b) une personne qui est déclarée coupable d'une telle infraction n'encourt à cet égard aucune autre peine que celle que prescrit la présente loi ou la disposition qui crée l'infraction.

La présomption d'innocence n'est pas pure abstraction; elle se traduit en pratique par des règles procédurales formelles issues du Common Law. Une de ces règles pratiques consiste par exemple dans l'obligation pour la poursuite de faire la preuve, hors de tout doute, de la culpabilité d'un accusé.

Concrètement, ce fardeau de la preuve permet en principe à l'accusé de garder le silence tout au long du procès et même de s'abstenir de présenter une défense s'il le juge opportun. La poursuite ne peut pas contraindre un accusé à témoigner dans son propre procès.

De même l'avocat de la poursuite ou le juge ne peuvent-ils commenter l'abstention de l'accusé de témo: oriłer ${ }^{3}$.

C'est à la poursuite de combattre la présomption d'innocence en établissant, par une preuve hors de tout doute, la culpabilité de l'accusé. Ce dernier n'a aucune obligation de convaincre ou de satisfaire le juge et le jury de quoi que ce soit ${ }^{4}$.

Ces règles reliées à la présomption d'innocence s'assouplissent toutefois au niveau de la sentence : la culpabilité établie, la présomption a été renversée. Ainsi l'accusé peut être interrogé par le tribunal sur certains aspects de sa personnalité; d'aucuns prétendent même qu'il pourrait, contrairement à ce qui prévaut au procès; être contraint à témoigner. Le droit au silence n'aurait plus sa justification lors de la sentence ${ }^{5}$. Cependant ces questions demeurent controversées en l'absence de texte législatif. En général on observe

3. Art. 4 (1) et 5, Loi sur la preuve au Canada, 1970, S.R.C., Chapitre E-10.

4. Exceptionnellement, l'accusé a un fardeau de persuasion explicite dans deux situations. Tout d'abord dans le cas de la défense d'aliénation mentale, c'est à l'accusé d'en faire la preuve par prépondérance de preuve. Puis, certains textes d'incrimination font reposer sur l'accusé la preuve d'une excuse légitime, d'une justification ou d'une autorisation. Outre ces cas spécifiques, il n'y a pas d'obligation de preuve pour l'accusé sauf bien entendu celle résultant de la stratégie judiciaire.

5. Voir l'exposé de ces questions dans Ruby, Clayton C. Sentencing. 2nd ed.. Butterworths, Toronto, 1981, p. 46; Tarasofsky, J. "Is the Accused A Compellable Witness Upon Sentence? "Thèse non publiée, Montréal, 1979; R. v. Butterwasser (1948), I A.B. 4. 
une réserve des tribunaux à mettre de côté, au stade de la sentence, les protections traditionnelles du procès contre l'auto-incrimination $^{6}$.

Cette dernière attitude des tribunaux illustrent un phénomène qu'on a généralement tendance à ignorer avec un haussement d'épaules. Il s'agit de ce qu'on pourrait appeler la philosophie du système ou si l'on préfère de l'atmosphère du procès.

En effet au-delà des objectifs spécifiques et immédiats de ces règles de droit, on perçoit qu'elles visent ultimement à une confrontation accusatoire équilibrée entre l'accusé et l'Etat. De cet objectif général se dégage une orientation qui se manifeste concrètement par un état d'esprit réel. Il s'agit du souci de ne pas causer préjudice à l'accusé. Cette préoccupation se présente tout d'abord comme une règle d'interprétation de dernier ressort. Il arrive en pratique que dans des situations limitrophes les juges ou les avocats utiliseront ce principe pour trancher une question en faveur de l'accusé.

De plus, le souci de ne pas causer préjudice à l'accusé devrait correspondre à une attitude générale du tribunal dans le règlement du déroulement des procédures. Si ce n'est pas toujours évident, il faut néanmoins constater l'existence d'une telle préoccupation chez plusieurs juristes.

En fait cet état d'esprit, ce «fair-play » du système contribue à balancer la confrontation judiciaire. Même si ce principe demeure une orientation, il justifie et légitime la répression des abus qui peuvent se manifester dans la conduite d'un procès. À titre d'exemple, la Cour d'appel a ordonné dans une affaire récente la tenue d'un nouveau procès à cause essentiellement des propos inflammatoires du réquisitoire de l'avocat du ministère public ${ }^{7}$.

Sur le plan des principes généraux, la règle de droit dans le procès apparaît non seulement comme le cadre de la confrontation mais aussi comme un frein et un contre-poids à l'abus et à l'arbitraire de la réaction sociale. Les principes juridiques ne peuvent prétendre régler tous les aspects, mais à y regarder de plus près ces derniers ne sont considérés comme abus et identifiés comme tels que par rapport à des standards juridiques qui constituent la recherche d'un idéal de justice. Que cela soit bien imparfait est l'évidence même, mais quiconque s'élève contre l'abus ou l'arbitraire pourra prendre au moins appui sur le principe du droit ou "Rule of Law » qui

6. Ainsi on a déjà jugé inapproprié pour un juge d'interroger directement l'accusé sur ses condạmnations antérieures; R. v. Donovan (1947), 4 C.R. 42.

7. Michel Dunn c. Sa Majesté la Reine (1981), IX R.D.P. 416; jugement prononcé le 27 avril 1981. 
apparaît à cet égard comme une sauvegarde fondamentale.

\section{LES SEQUENCES DU PROCES}

\section{Le déclenchement du processus judiciaire}

Sur le plan strictement juridique, le procès débute par la dénonciation. Plusieurs n'y voient qu'une simple formalité et s'étonneront peut-être qu'on s'y intéresse. Pourtant cette "formalité » est essentielle et son absence enlève toute légalité aux mesures judiciaires ou autres prises subséquemment contre l'accusé. Une détention sans dénonciation, donc sans comparution, est illégale.

Plus encore, la dénonciation est une application particulière du principe de la légalité. Elle consiste dans l'énoncé formel de l'infraction commise par une personne en indiquant les détails factuels et juridiques qui permettent d'identifier l'acte reproché et son auteur. Elle informe donc l'accusé et publicise la confrontation.

Dans le processus judiciaire c'est un premier filtre juridique et administratif. La dénonciation se fonde sur les allégations d'un dénonciateur reçues et vérifiées par un juge de paix ${ }^{8}$. Cette "passoire " est le filtre prévu par la loi. Il s'agit donc uniquement de la vérification sommaire de la correspondance entre un accusé, des faits et une accusation. C'est peu, mais essentiel et il semble qu'à ce niveau des procédures, on a tenu suffisamment compte du peu de conséquences immédiates pour l'accusé.

Toutefois, il peut se glisser des erreurs, la dénonciation peut être faite par malice ou fanatisme, la preuve recueillie peut ne pas justifier l'accusation. Aussi, le ministère public du Québec a-t-il institué depuis quelques années une procédure administrative supplémentaire qui suscite d'ailleurs un vif intérêt des organismes de poursuite des autres provinces canadiennes.

Au Québec, toute plainte au criminel qu'elle émane des corps policiers ou d'un plaignant privé doit faire l'objet d'un examen par un substitut du procureur général. Non seulement l'accusation y est-elle vérifiée mais aussi la preuve et l'opportunité de la poursuite. Un des avantages de cette procédure est l'élimination systématique des dossiers où l'accusation serait sans fondement juridique ou factuel. Cette procédure a permis également de faire libérer, avant comparution, certaines personnes accusées de bris de conditions. En effet, il arrive parfois que la condition enfreinte ne s'applique plus à

8. Cette vérification se fait soit par simple lecture des allégations soit par l'audition de témoins lors de la tenue d'une pré-enquête. Cf. art. 4553 (1) et 455.3 (3) C. Cr. Sur la dénonciation même, cf. 455 et 724 (1) C. Cr. 
l'accusé. L'erreur, d'ordre technique, provient d'un mauvais acheminement de l'information dans les banques de données accessibles aux corps policiers.

Des cas plus graves, mais heureusement plus rares peuvent également être repérés dès le départ. Le refus d'autoriser la plainte permet ainsi d'empêcher une accusation injuste. Par exemple, il arrive qu'à la suite d'une arrestation, par ailleurs légale, il n'est rien qui puisse être reproché à un individu. Normalement, les policiers relâchent la personne et bien sûr ne présentent pas de dénonciation puisqu'aucun fait ne la justifie. Les policiers ont non seulement la discrétion mais aussi le devoir d'agir de la sorte. On a déjà tenté, toutefois, de présenter de semblables dénonciations non fondées et ainsi faire entreprendre des procédures injustes contre des individus. L'inexpérience et la crainte des supérieurs, le zèle administratif $^{9}$ ou encore la crainte d'une poursuite pour arrestation illégale ont parfois conduit à de telles attitudes. Fort heureusement ces quelques cas ont pu être stoppés à temps grâce au contrôle du procureur général, mais malheureusement il en est d'autres qui réussissent.

Même s'il s'agit de cas exceptionnels sur le volume de dossiers conduits par les forces de l'ordre, ces quelques exemples peuvent apparaître comme les symptômes d'un certain malaise chez les policiers. Malaise relié d'abord à la définition de leur rôle en particulier qui est traditionnellement axé sur la répression, même lorsqu'il s'agit de prévention. De plus, une certaine négation, par différents milieux, de la discrétion qui peut être exercée par le policier, rend celui-ci hésitant à l'exercer. Ces pouvoirs de discrétion ne sont évidemment pas absolus ni très étendus, mais ils sont mal connus ou mal appliqués. C'est aussi une question de jugement dans leur utilisation $^{10}$.

La plainte autorisée, la dénonciation est présentée au juge de paix pour la vérification prévue par la loi. Il faut noter qu'il y a toujours moyen pour un dénonciateur de contourner la vérification administrative. Après un refus d'autorisation par un substitut, on peut par exemple se présenter devant un autre procureur en se gardant bien de lui parler $d u$ refus précédent. On peut même se conformer strictement aux dispositions du Code criminel et présenter la dénonciation directement au juge de paix tout en taisant les mésaven-

9. Une de ces tentatives a déjà été expliquée comme une nécessité statistique. C'est plutôt maladroit mais tristement de bonne foi.

10. Les causes de responsabilité civile des policiers sont assez éloquentes à ce sujet. Voir entre autres Chartier c. Le Procureur général du Québec (1979), 2 R.C.S. 475. 
tures antérieures. Le procédé n'est pas une garantie de succès, mais il réussit souvent. Si ce n'est pas très honnête, ce n'est pas illégal. L'odieux de la stratégie réside évidemment dans les motifs du dénonciateur et aussi dans le mépris de l'opinion émise.

Il y a là une lacune dans la loi. Le Code criminel est en effet silencieux quant à l'intervention du procureur général à ce niveau. Ceci est d'autant plus étonnant que juridiquement cette intervention procède, entre autres, de la discrétion première qui est dévolue en Common Law au procureur général en matière de décision de poursuivre ou non ${ }^{11}$.

Quoi qu'il en soit l'emploi de ce genre de "technique " n'est pas limité à la dénonciation et aux dénonciateurs. Bien que la chose soit plus difficile de nos jours à cause du système de confection des rôles, les avocats tentent de choisir « leur " juge pour faire une cause ou enregistrer un plaidoyer. Mais les politiques strictes des cours en matière de remise empêchent en pratique ce "shopping" qui était un véritable problème il y a quelques années.

La dénonciation est suivie de l'émission d'une sommation ou d'un mandat d'arrestation afin d'assurer la comparution de l'accu$s^{12}$. Cependant lorsqu'il y a arrestation sans mandat la dénonciation se fait généralement peu avant la comparution. Toutes ces procédures sont réglementées par la loi qui prévoit un cadre relativement strict d'utilisation. Ce qui n'est pas prévu cependant c'est la façon dont ces procédures, particulièrement l'arrestation, sont utilisées.

Certes le droit précise les cas où il peut y avoir arrestation ${ }^{13}$ et même une règle de prudence afin d'éviter l'arbitraire. Cependant cette règle, fondée sur les motifs raisonnables et probables qui doivent fortifier la conviction de l'agent de la paix, demeure bien subjective et constitue un bien faible rempart contre l'arbitraire. Toute suspicion peut être fondée sur un motif raisonnable et probable. Ce que les policiers eux-mêmes appellent "l'enquête de routine " offre l'exemple type d'un certain laxisme dans l'interprétation d'une règle de droit. Il est vrai que de pures enquêtes de routine se révèlent par la suite avoir été des arrestations fondées, mais pour les autres?

Les cas que la jurisprudence nous donne d'arrestations illéga-

11. Pour apprécier la portée des prérogatives du procureur général voir entre autres l'affaire Pierre Morneau c. Joseph Rodriguez, décision non rapportée du 27 janvier 1976 (no 38-000004-76-1, C.S.).

12. Art. 455.5 et 728 C. Cr. et 456 (2) C. Cr.

13. Art. 449,450 et 451 C. Cr. 
les ou encore mieux les cas d'acquittement pour une accusation d'entrave à un agent de la paix ${ }^{14}$, dévoilent des pratiques qui nous conduisent aux confins de la légalité. Les abus qu'il peut y avoir au niveau de l'arrestation ne sont que le reflet d'un problème social beaucoup plus généralisé et fondamental.

Le procès commence dans la rue. Le policier qui applique la loi pénale est appelé à poser des jugements qui nécessairement se fondent sur sa perception des choses, sur ses propres valeurs et aussi sur ses conceptions personnelles de l'ordre. C'est ainsi qu'il véhicule, généralement inconsciemment, un système de valeurs auquel il associe étroitement le code pénal. Les marginaux en font les frais! Encore là il ne faut pas généraliser la proposition, mais il est probable que l'application simultanée de la loi et du système de valeurs dominantes fausse la perspective strictement juridique du code ${ }^{15}$.

Aussi, tout ce qui ne cadre pas avec ce système de valeur devient suspect. Dans certains milieux populaires où l'indice de criminalité est plus élevé, du moins pour les crimes de brigandage, la suspicion peut se transformer en véritable harcèlement.

Plusieurs facteurs contribuent à cet état de choses. Tout d'abord le milieu criminel véritable, lui-même. On n'a qu'à lire quelques dossiers pour constater qu'il existe des familles entières où la criminalité est un mode de vie qui se transmet d'une génération à l'autre. De plus les criminels actifs d'un quartier sont souvent connus des policiers qui suivent leur évolution depuis l'enfance. Cette situation explique, mais ne saurait en aucun cas justifier, l'application arbitraire de la loi pénale. Des témoignages d'individus affirment qu'uniquement parce qu'ils sont connus des policiers ils font régulièrement l'objet d'enquêtes injustifiées et parfois même gratuites. Tel autre peut être arrêté pour "fins d'interrogatoire", non qu'on ait quelque chose de précis à lui reprocher, mais parce qu'il a un frère ou un ami qu'on suspecte. On pourrait compléter ce tableau sur une infinité de petits détails qui sans trop causer individuellement de préjudice réel finissent par créer une certaine injustice.

Au niveau de la prévention et du dépistage du crime, la règle de droit étant moins présente qu'au procès, le contrôle judiciaire étant absent, l'arbitraire se développe plus librement. Ses manifesta-

14. Art. 118 C. Cr.

15. La création de corps policiers "autochtones " atteste de la reconnaissance, ou tout du moins de la réalité du phénomène sur le plan culturel. Qu'on songe par exemple aux policiers amérindiens, noirs et même homosexuels à San Francisco. 
tions ne sont pas limitées au seul milieu populaire, elles se retrouvent à l'égard d'autres groupes minoritaires : les adolescents, certains groupes ethniques, etc.

Dépendant du milieu, une allure originale ou une démarche particulière éveille plutôt l'attention. Un jeune transportant un sac sur la rue à une heure tardive devient plus aisément un suspect de choix. À cause de sa perception du milieu, l'intervention du policier se fait plus facilement. Les motifs raisonnables et probables sont déjà trouvés. Ce comportement psychologique et social des agents de la paix est généralement inconscient. Ses causes sont multiples. Le dépit. la facilité de la routine et l'absence d'introspection par rapport à ses propres valeurs expliquent en partie l'attitude. Les conséquences injustes n'en sont pas moins réelles.

Dans certains cas, elles prennent des proportions inquiétantes. On peut observer des situations où à la suite d'une arrestation illégale, ou du moins injustifiée, on cherche la moindre peccadille pour porter une accusation et justifier ainsi rétrospectivement l'intervention. Pire encore, il est des affaires judiciaires où il apparaît qu'on a provoqué ou facilité une réaction violente de l'individu arrêté. L'accusation d'entrave ou de voies de fait sur un agent de la paix sanctionne alors le phénomène. Ces abus, lorsqu'ils sont connus, sont réprimés. Par contre, ils accroissent le climat de tension entre les policiers et certains milieux, en particulier le milieu strictement criminel, ce qui ne peut être que générateur de violence. L'escalade est évidemment facile et la violence policière est loin d'être mythique. Non pas qu'il n'y ait pas une violence exercée à l'encontre des policiers. Elle existe. Mais les deux sont injustifiées et contraires au Droit.

Progressivement la violence peut s'infiltrer sournoisement dans les moeurs des policiers. La lutte contre la criminalité devient alors presque une chasse aux criminels. Ce n'est pas exagération. Je me rappelle un cas où l'accusé, un père de famille à sa première expérience criminelle (et probablement la dernière) a éclaté en sanglots en admettant sa culpabilité devant le tribunal. Exprimant des remords pour son acte, il a précisé que l'atteinte à la dignité humaine qu'il avait subie et l'impuissance qu'il avait ressentie lors de son arrestation l'avaient blessé irrémédiablement. Il avait été giflé et insulté par un policier chez lui devant ses enfants.

La violence policière existe mais elle est bien camouflée. Nous nous en faisons les complices par le silence gardé autour d'un phénomène qu'on perçoit comme un mal nécessaire. Et puis n'est-elle 
pas exercée contre des criminels, des gens eux-mêmes violents et par définition peu crédibles? Enfin les difficultés de la preuve, les priorités de la lutte contre le crime et l'indifférence surtout finissent par faire accepter l'intolérance et la violence. Encore une fois il ne faut pas généraliser, mais les quelques cas qui se manifestent appellent la vigilance.

Du point de vue du principe de la légalité, le traitement du prévenu à la suite de son arrestation n'est guère plus reluisant. Les seuls contrôles juridiques entre le moment de l'arrestation et la comparution de l'accusé en cour se résument dans le délai de vingtquatre heures dans lequel doit se faire la comparution, et dans les quelques règles d'admissibilité en preuve des déclarations que peut faire un individu en état d'arrestation. Le droit au silence et le droit d'avoir un avocat sont bien sûr respectés, mais dans des conditions suspectes.

La règle de droit exige que pour retenir les aveux d'un accusé faits aux policiers, il faut que sa déclaration ait été faite librement et volontairement, sans promesses ni menaces de la part de ceux qui l'ont recueillie. La Cour Suprême du Canada dans une série de jugements récents ${ }^{16}$ a précisé, en la réduisant, la notion de déclaration libre et volontaire : il faut que ce soit "l'expression d'un esprit conscient ". En pratique ceci veut dire que le traumatisme ou l'état de choc que peut avoir un accusé l'empêche de faire une déclaration libre et volontaire et ce, même s'il n'y a eu aucune promesse ni menace de la part des policiers. Il apparaît significatif que la Cour Suprême limite ainsi la notion d'aveu libre et volontaire.

D'ailleurs en général, l'approche juridique de l'aveu est contradictoire. D'une part l'aveu est la « reine des preuves » et d'autre part on exige d'elle des caractéristiques qui cadrent plus ou moins avec la réalité psychologique.

Il ne saurait être question dans ce texte de passer en revue les " techniques » connues d'obtention d'une déclaration. Une chose demeure cependant, les conditions générales d'obtention des aveux qui ressortent lors d'un voir-dire sont d'un stéréotype suspect. L'absence de contrôle externe, l'étanchéité du poste de police et les échos de diverses sources qui nous parviennent n'aident pas à dissiper le doute quant au respect intégral de la règle de droit. Enfin

16. Voir les arrêt Ward v. The Queen, (1979) 2 S.C.R. 30; Nagotcha v. The Queen, (1980) v. R.D.P. 655; 1 B.C.R. 714; Rothman c. Sa Majesté la Reine (1981) VIII R.D.P. 545; jugement prononcé le 2 mars 1981 et Horwath v. The Queen (1979) 2 S.C.R. 376. 
quand on sait que la majorité des dossiers contiennent des déclarations et que même dans quelques-uns d'entre eux il s'agit de l'essentiel de la preuve et de l'enquête policière, comment ne pas s'étonner de la «spontanéité » des accusés.

\section{Les procédures préliminaires}

La comparution est la première apparition formelle du prévenu devant la cour.

L'article 454 (1) prévoit qu'une personne qui a été arrêtée et est détenue doit comparaître dans les 24 heures de l'arrestation à moins qu'aucun juge de paix ne soit disponible durant cette période. Dans un tel cas, la comparution doit avoir lieu le plus tôt possible.

Le détenu peut alors subir une enquête sur la question de sa mise en liberté en attendant son procès.

Cette enquête a lieu devant le juge de paix de la comparution à moins qu'il ne s'agisse d'un des crimes de l'art. 457.7 pour lesquels seul un juge de la Cour supérieure a compétence pour tenir une telle enquête.

À l'enquête sur la mise en liberté, le code suggère l'orientation de base en matière de détention. Celle-ci doit être l'exception.

Ainsi la mise en liberté est la règle à moins que le poursuivant ne s'y objecte. Dans certains cas toutefois, le fardeau d'obtenir la remise en liberté repose sur le prévenu.

Il peut y avoir révision de la décision du juge de paix à l'enquête. Cette révision a lieu devant la Cour Supérieure (457.5; 457.6 C. cr.)

À l'issue de la comparution le juge de paix pourrait tenir, en principe, une enquête préliminaire (dans le cas d'actes criminels), cependant en pratique il ajourne la cause à une date déterminée pour la divulgation de la preuve et l'enquête préliminaire ou le procès. (465 (1) C. cr.).

La divulgation de la preuve a pour objectif de réduire le volume des causes par la recherche d'accords entre avocats quant à certaines admissions de faits non litigieux.

L'attitude vis-à-vis de ce procédé varie énormément. À l'enthousiasme de certains juristes s'oppose la farouche hostilité des autres. Si bien que la procédure prend des aspects différents d'un dossier à l'autre. Les avocats aussi bien de la poursuite que de la défense hésitent parfois à admettre ce qui est perçu comme une brèche dans leur stratégie. En effet, certains profiteront toujours de l'occasion de l'absence d'un témoin pour demander la libération de l'accusé. Faire des admissions préalables c'est renoncer à cette possibi- 
lité. D'un autre côté demander à la Couronne de dévoiler toute sa preuve sans contre-partie d'admission c'est priver la procédure de divulgation de son objectif et de son utilité.

Mais au-delà de ces considérations premières, c'est la philosophie même du système accusatoire tel qu'il est pratiqué qui est remise en question. En effet, si on excepte ses aspects strictement juridiques, le travail de l'avocat lors d'un procès consiste en grande partie dans la stratégie. Tout est mis en cuvre pour gagner une cause : la personnalité même de l'avocat, les effets surprises, l'atmosphère du débat, etc. Pour certains, sans scrupules intellectuels, tous les coups sont permis. Ainsi un avocat de la défense ayant une expérience de plusieurs années n'hésitera pas à tabler sur l'inexpérience d'un jeune procureur de la poursuite plutôt que sur son "bon droit ». On cherche à prendre l'autre en défaut, à trouver la faille, moins dans la preuve que dans les erreurs que l'adversaire peut commettre ou qu'on a provoqué expressément à l'occasion. Tout cela est bien loin de la règle de droit, mais considéré de bonne guerre. On peut s'interroger.

À ces aspects stratégiques s'ajoutent plusieurs épiphénomènes qu'il serait trop long d'examiner ici dans les détails. Qu'il suffise cependant de signaler que les attitudes psychologiques qu'on observe dans le quotidien se retrouvent évidemment dans le déroulement d'un procès. Les préjugés sociaux malgré les balises du droit peuvent influer sur une décision judiciaire lorsqu'il y a une certaine marge discrétionnaire accordée par la loi. Les avocats eux-mêmes en conviennent qui catégorisent parfois les juges en "favorables à la défense " ou en "favorables à la Couronne ». Ce genre de phénomène est certes superficiel mais néanmoins réel.

Les apparences aussi peuvent jouer un rôle déterminant. L'attitude d'un témoin ou de l'accusé, la sympathie ou l'antipathie qu'ils inspirent, la nature même du crime jugé représentent autant d'éléments qui contribuent à la formation du jugement.

Ces épiphénomènes sont les parasites du processus judiciaire dont ils font cependant partie intégrante. Le droit tente d'en limiter les effets négatifs au nom d'un idéal de justice qui est peut-être voué à demeurer un idéal. Comme le disait fort à propos le juriste américain Cardozzo, la justice demeure une justice d'hommes. Cependant, là oủ l'injustice apparaît flagrante il est indispensable de la réprimer. 


\section{L'instruction ou le procès}

Le procès pour acte criminel débute par la mise en accusation qui est la présentation formelle d'un acte d'accusation devant le tribunal chargé d'instruire la cause. Pour l'infraction sommaire, le procès s'instruit tout simplement sur la base de la dénonciation originelle.

Le procès lui-même consiste principalement dans la présentation de la preuve. Les grandes étapes de l'instruction peuvent se résumer comme suit :

Preuve principale de la poursuite. - Le poursuivant présente la preuve qui soutient l'accusation. Celle-ci est généralement constituée par les déclarations des témoins que le représentant du ministère public interroge et que peut contre-interroger la partie adverse. C'est souvent l'occasion pour l'avocat de faire valoir sa personnalité. Parfois ce succès personnel est alors un élément plus fort que le triomphe du droit.

La poursuite doit établir l'existence de tous les éléments de l'infraction reprochée à l'accusé et démontrer hors de tout doute raisonnable la participation de celui-ci à la perpétration du crime.

Lorsque les témoins à charge ont été entendus, la poursuite déclare sa preuve close.

La motion de non-lieu et la réouverture d'enquête. - Si la poursuite a fait défaut de prouver un des éléments essentiels de l'inculpation, l'accusé pourra présenter une motion de non-lieu devant juge seul, ou une requête pour verdict dirigé devant jury, et ainsi obtenir un acquittement.

Le ministère public peut cependant obtenir dans certains cas, une ré-ouverture d'enquête qui lui permette de compléter sa preuve et ainsi faire échec à la motion de non-lieu.

Si la requête de la défense est rejetée, le procès doit alors continuer. Par contre, si la requête est acceptée, le juge libère aussitôt l'accusé.

Preuve principale de l'accusé. - L'accusé peut avoir des témoins à faire entendre ou témoigner lui-même en défense. L'avocat de la poursuite peut alors non seulement contre-interroger ces témoins mais aussi l'accusé qui témoigne volontairement dans sa cause. C'est uniquement à cette occasion qu'un accusé pourra se voir interrogé sur ses antécédents judiciaires par la poursuite qui teste ainsi la crédibilité de l'accusé-témoin.

La contre-preuve. - Lorsque l'inculpé a présenté sa défense et déclaré sa preuve close, le poursuivant peut entreprendre une 
contre-preuve pour réfuter les arguments de son adversaire, ou infirmer la valeur probante des faits nouveaux que ce dernier a pu révéler dans sa défense.

La réplique. - L'accusé peut répliquer à son poursuivant par sa contre-preuve chaque fois que celui-ci a apporté des faits nouveaux et chaque fois que l'intérêt de la justice le requiert.

Les plaidoinies et l'adresse du juge au jury. - Lors des plaidoiries devant juge seul, les parties discutent des faits de la cause et des points de droit qu'elle implique. Devant une cour composée d'un juge et d'un jury, les avocats se contentent de récapituler la preuve et d'analyser les faits qu'elle a révélés.

C'est souvent l'occasion pour les avocats d'exercer leur talent de comédiens. Ils n'y manquent pas. Un ex-juré me confiait un jour que la personnalité et le jeu théâtral de l'avocat contribuait pour beaucoup au sort d'une cause.

Dans le cas d'un procès par jury, c'est le jury qui est le " maître des faits " et qui rend le verdict final sur la culpabilité ou l'innocence de l'accusé. Le juge doit alors s'adresser aux jurés afin de leur faire comprendre leur rôle et leur expliquer les décisions qu'ils peuvent rendre. L'adresse du juge au jury revêt une grande importance puisqu'il explique aux jurés la loi applicable, les arguments soulevés par l'accusé, les éléments de preuve apportés par la poursuite et les questions sur lesquelles ils doivent rendre le verdict.

Le verdict. - Lorsque le tribunal est composé d'un juge et d'un jury, c'est le jury seul qui se prononce sur l'innocence ou la culpabilité de l'accusé. Au Canada, le juge ne délibère pas avec le jury et celui-ci n'a pas à se préoccuper de la sentence.

Le verdict de culpabilité ou d'acquittement prononcé par un jury doit être unanime (art. 580 C. Cr.).

Lorsque le tribunal est composé d'un juge sans jury c'est évidemment le magistrat seul qui prononce à la fois le verdict et, s'il y a lieu, la sentence.

\section{La sentence}

Lorsque à l'issue du procès ou à la suite d'un plaidoyer un verdict de culpabilité a été prononcé, se pose alors la question de la sentence.

Quels que soient les délits commis et la procédure appliquée pour en poursuivre l'auteur l'imposition de la peine relève toujours du juge qui préside le tribunal. 
Le Code lui accorde d'ailleurs en ce domaine, une discrétion très importante, limitée uniquement par la légalité des sanctions pour un crime particulier.

La phase judiciaire du traitement de la criminalité se termine généralement par l'imposition de la sentence. Certes, le bris d'ordonnance peut faire l'objet d'une intervention qui ramène l'accusé devant le tribunal mais il s'agit là d'une mesure de contrôle relativement isolée dans l'ensemble du domaine sentenciel. En fait, contrairement à certains autres systèmes judiciaires, le domaine de l'application des peines relève plutôt de l'administration que du judiciaire. On peut s'interroger cependant sur la nécessité d'une présence plus sensible du judiciaire dans ce domaine au cours des prochaines années.

\section{CONCLUSION}

Comment conclure ce rapide survol d'un sujet ayant l'ampleur de celui que nous avons décidé d'aborder dans ce texte? Les contraintes d'espace et de temps nous empêchent malheureusement de pousser plus loin notre analyse. Cependant, celle-ci est loin d'être terminée car chaque phase de procédure est susceptible d'offrir des dessous inattendus, Le procès se révèle être plus qu'une simple institution juridique; c'est également un phénomène de société avec toutes les contradictions et toutes les luttes que cela comporte, mais aussi avec tous les espoirs et une certaine recherche d'un idéal de justice.

\section{BIBLIOGRAPHIE SOMMAIRE}

CROSS, R. \& ASTERLEY (1968) : An Introduction to Criminal Law, 6th edition, London, Butterworths.

FALLON, P. (1975) : Crown Court Practice : Sentence, London, Butterworths.

FALLON, P. (1978) : Crown Court Practice : Trial, London. Butterworths.

FORTIN, J. (1971) : L'Infraction, Montréal, Presses de l'Université de Montréal.

GLAZEBROOK, P.R. (ed) (1978) : Reshaping the Criminal Law Essays in Honour of Glanville Williams, London, Stevens \& Sons.

HALSBURY, (1976) : Halsbury's Laws of England, 4th edition by Lord Hailsham of St-Marylebone (Vol. II), London, Butterworths.

LAGARDE, I. (1962) : Droit pénal canadien, Montréal, Wilson et Lafleur.

MEWETT, A.W. \& MANNING, M. (1978) : Criminal Law, Toronto, Butterworths.

PARIZEAU, A. et SZABO, D. (1977) ; Le traitement de la criminalité au Canada. Montréal, Presses de l'Université de Montréal.

RUBY, C.C. (1981) : Sentencing, 2nd edition. Toronto, Butterworths. 
SAlHaNY, R.E. (1978) : Canadian Criminal Procedure, 3rd edition, Toronto, Canada Law Book.

WOOTTON, B. (1963) : Crime and the Criminal Law, London, Stevens \& Sons.

Rapports et Études de la Commission de Réforme đu Droit du Canada:

- La crainte du châtiment : la dissuasion.

- La participation communautaire à la réadaptation du délinquant.

- Sentences et mesures non sentencielles dans le processus pénal.

- Études sur le Sentencing. 